\title{
Tendencias y retos en la gestión de organizaciones deportivas: Perspectivas en Colombia
}

\section{Trends and challenges in the management of sport organizations: Perspectives in Colombia}

\author{
Liliana María Cardona Mejía* y Santiago Cardona Ramírez
}

Universidad de Antioquia (Colombia).

\begin{abstract}
Resumen: Este estudio tuvo como objetivo reconocer las tendencias y retos en la gestión de organizaciones deportivas públicas y privadas de Colombia. Para ello, se desarrolló un análisis teórico desde la literatura alrededor del tema y un análisis de contenido con información que se aportó en un panel de reconocidos expertos en el área. Los resultados señalan la innovación, la incorporación de tecnologías de la información y la comunicación, la gestión del cambio organizacional, la necesidad de fortalecer los perfiles de formación de los dirigentes, el relacionamiento interinstitucional y el trabajo en redes, como algunos de los retos más importantes para los directivos. Constituye un punto de partida para el diseńo de planes en las organizaciones y planes de formación y capacitación para directivos.

Palabras clave: Tendencias, retos, gestión de organizaciones deportivas.
\end{abstract}

Abstract: This study aimed to recognize trends and challenges in the management of public and private sport organizations in Colombia. We realized a theoretical analysis from the literature around the subject and a content analysis with information provided in a panel of recognized experts in the area. The results indicate innovation, the incorporation of information and communication technologies, the organizational change management, the need to strengthen the training profiles of managers, inter-institutional relations and networking, as some of the most challenging important for managers. It is a starting point for the design of plans in organizations and plans of training for managers.

Keywords: Trends, challenges, management of sport organizations

\section{Introducción}

La administración deportiva es un área en creciente desarrollo que demanda cada vez más la mirada de académicos e investigadores en aras de generar rutas y estrategias para gestionar adecuadamente las organizaciones. Conocer las tendencias y retos que a partir de las demandas del entorno surgen, se convierte en un asunto imprescindible en las organizaciones. Este estudio tuvo, por tanto, el objetivo de reconocer dichas tendencias y retos a partir de la información que aportaron reconocidos expertos en Colombia en la dirección de organizaciones deportivas. Se presenta una perspectiva que, en cualquier caso, demanda la habilidad del directivo para hacer un adecuado análisis y elegir las estrategias que respondan de mejor manera a dichas demandas.

Reconociendo que la literatura de impacto sobre este tema es escasa en Colombia, las voces de expertos se convierten en un insumo importante que permite delinear un marco teórico inicial a partir del cual pueden generarse otros desarrollos. En este sentido, este artículo presenta un panorama frente a las tendencias y retos en la gestión de organizaciones deportivas a partir del análisis que se ha hecho

Dirección para correspondencia [Correspondence address]: Liliana María Cardona Mejía. Universidad de Antioquia (Colombia).

E-mail:1maria.cardona@udea.edu.co de las intervenciones de un panel de expertos en administración deportiva.

\section{Revisión de literatura}

Diversas profesiones y contextos utilizan el término tendencia, ligado siempre a una invitación de cambio. Al hablar de tendencias se hará alusión a las corrientes, direcciones, prospectivas, cursos y acontecimientos (López, 2015; Ogburn, 1957) que para el caso del presente trabajo, surgen en la gestión de organizaciones deportivas. Esto es, aquellas acciones que se vienen desarrollando en el contexto de dichas organizaciones y que debieran ser tenidas en cuenta por sus directivos, desde la perspectiva de retos y cursos de acción, para estar a la vanguardia. En este sentido, una tendencia genera a su vez un reto en la organización, en el interés de responder y adaptar las prácticas organizacionales a dichas tendencias. Dada esta relación, el análisis de tendencias y retos en las organizaciones, se consideró de manera conjunta.

Indudablemente la globalización y el auge de las tecnologías de la información y la comunicación han impulsado una serie de retos para las organizaciones (Bustelo-Ruesta y García-Morales-Huidobro, 2001; Herrera, 2008). Los aparatos tecnológicos, por ejemplo, se han convertido en uno de los principales medios de comunicación en la sociedad y son 
usados por millones de usuarios (López-Carril, Villamón et al., 2019). Acontecimientos de este tipo obligan a las organizaciones e instituciones a implementar procesos de cambio permanentemente, por tanto, uno de los retos actuales para los directivos es aprender a gestionar el cambio organizacional adecuadamente, identificando facilitadores, reduciendo resistencias y desarrollando conscientemente cada una de sus fases: preparación, cambio en sí mismo e implementación (Cardona, 2017).

En el afán de sobrevivir en un mercado que se hace cada vez más competitivo, diversos modelos de gestión son aplicados, de manera intencionada o no, en la gestión de organizaciones, tales como el normativo, estratégico, burocrático, calidad, entre otros (Casassus, 2000; Zaratiegui, 1999 ). Un enfoque que ha tomado fuerza y se convierte en tendencia es la gestión de la calidad y ligada a ella, la gestión por procesos centrando su interés en el liderazgo, el enfoque al cliente, la mejora continua y la toma de decisiones (Herrera, 2008; Pires y Machado, 2006).

Las organizaciones del deporte no son ajenas a esta realidad, dado que funcionan desde la misma lógica de cualquier otra organización o institución. Tienen una estructura, unos recursos y capacidades implicados, planes, proyectos y objetivos en común, por tanto, las teorías, principios y estrategias organizacionales que se desprenden de los estudios en administración y gestión, son aplicables a ellas (Cardona y Padierna, 2017; Escobar, 2011; Fernández-Alles y ValleCabrera, 2006).

En este sentido, se retoma también el planteamiento de Cardona, Padierna, Córdoba y González (2013) que asumen la administración deportiva como una rama de la administración general, poniéndose en escena la habilidad de los directivos para aplicar sus principios y teorías al contexto específico de la organización donde se desempeñan. Es necesario mencionar que la gestión del deporte no logra consolidarse como un campo de estudio específico -exceptuando el sport management que goza de amplia tradición y trayectoria- dado que la producción científica es aún escasa (Cardona, 2013; López-Carril, Añó y Villamón, 2019), por tanto, es otro gran reto que se enfrenta en las instituciones con el interés de promover la formación de investigadores en el área.

En el escenario específico de organizaciones deportivas, un estudio llevado a cabo por la Universitat Pompeu Fabra (2017), plantea cinco retos para la industria del deporte, como son la búsqueda de nuevas formas de patrocinio que sean creativas y generen alianzas, crecimiento y sostenibilidad económica, gestión y uso adecuado de información y tecnologías, adquisición de plataformas deportivas que permitan mayor interacción con seguidores a través de redes sociales y la aceptación, regulación y consolidación de los e-sports. Un panorama similar presentan López-Carril, Villamón et al. (2019) quienes reconocen que los medios sociales se presen- tan hoy como un gran reto para la industria del deporte, dado que son una estrategia de visibilización, divulgación, posicionamiento, interacción con la comunidad y, en tal sentido, deben hacer parte de la formación de un dirigente deportivo en el afán de lograr incorporarlos a su organización y gestionarlos adecuadamente. Esto es un llamado también para las instituciones de educación superior para que incluyan este componente en sus planes de formación.

Chalip, citado por López-Carril, Añó y Villamón (2019), dan cuenta de la evolución que ha tenido el deporte, destacando su contribución a la salud, la socialización, el crecimiento económico, el desarrollo de la comunidad y, dados los resultados de los atletas que representan a los países, el orgullo nacional. Zeimers et al. (2019) mencionan algunas características que dan cuenta de la transformación que han tenido las organizaciones deportivas y que generan retos sobre su gobernanza, tales como las relaciones con stakeholders, las alianzas y redes interinstitucionales, la tensión que existe entre el deporte de élite y el deporte para todos, entre otros.

También se expresan retos relacionados con el crecimiento de las organizaciones desde los medios de comunicación, el empoderamiento de los usuarios, procesos de capacitación, profesionalización de la gestión, redes de trabajo, financiación, innovación, calidad, nuevos modelos de gobernanza, entre otros (Da Pena \& Fierli, 2017). Estos retos son reconocidos por Tortora (2018), sin embargo, resalta la necesidad de crear nuevos modelos de negocio a partir de los cambios acelerados del entorno, dando respuesta a los altos estándares de calidad que hoy demandan los usuarios de servicios de actividad física y deporte. Esto hace que la innovación se convierta en un tema esencial en la gestión de organizaciones deportivas.

Otros retos expresados por Escobar (2011) en el contexto colombiano, tienen que ver con la formación en administración de los dirigentes deportivos y la creación de un marco legal que regule el perfil y las formas de elección. En este mismo sentido, el estudio de Padierna et al. (2019) presenta un panorama desalentador en torno a la formación de dirigentes deportivos y, de manera específica, de gestores de instalaciones deportivas, dado que solo el $43 \%$ cuenta con formación profesional, un $4 \%$ tiene formación posgradual y, en algunos casos, los gestores ni siquiera tienen estudios de educación superior o terciara. Por supuesto, esta situación limita las posibilidades de desarrollo del sector y el funcionamiento de las organizaciones bajo la premisa que se ha establecido, desde modelos, principios y teorías de la administración. Un reto para las instituciones, tiene entonces que ver con la generación de oportunidades de formación profesional y posgradual para los dirigentes actuales y futuros, de manera tal que desarrollen habilidades directivas que les permitan hacer adecuados diagnósticos y lecturas del entorno y seleccionar las estrategias de gestión que mejor se ajusten a su contex- 
to y conduzcan al logro de los objetivos organizacionales. A propósito, el trabajo de Padierna et al. (2019) presenta una propuesta de contenidos para la formación disciplinar y permanente de gestores deportivos.

A partir de estos hallazgos de la literatura, se han identificado y agrupado retos en la gestión de organizaciones, que tienen que ver con:

- La innovación, el marketing y los nuevos modelos de negocio

- Las tecnologías de la información y la comunicación, las redes sociales, los e-sports

- La contribución del deporte y la actividad física al bienestar de las comunidades desde lo social, lo económico y la salud.

- La necesidad de formación gradual y posgradual para dirigentes deportivos y la buena gobernanza

- La articulación entre organizaciones del sector y el trabajo en redes

Dichos hallazgos son el soporte teórico para el análisis de contenido que se hace en el presente trabajo, intentando compararlos con las aportaciones que realizaron los sujetos indagados como expertos en la administración y gestión de organizaciones deportivas.

\section{Metodología}

Se ha desarrollado un análisis de contenido a partir de las orientaciones de Krippendorff (1990) que lo reconoce como una técnica de investigación científica que permite comprender significados, mensajes, funciones y efectos desde unos datos esencialmente verbales, simbólicos o comunicativos, a partir de la construcción de inferencias. Para este caso, los datos se originan desde un panel de expertos en gestión de organizaciones deportivas, en el marco del Foro: tendencias contemporáneas en la gestión de organizaciones del deporte, desarrollado en la región de Antioquia, Colombia. El panel fue integrado por tres expertos, de la siguiente manera:

- Un directivo de organización deportiva nacional, con amplia experiencia en la dirección de organizaciones en el ámbito nacional, departamental y municipal

- Un directivo en el ámbito departamental, con amplia experiencia en la dirección de eventos deportivos nacionales e internacionales

- Un directivo en el ámbito municipal, con experiencia en la dirección y gestión de instalaciones y equipamientos deportivos

El procedimiento consistió en el planteamiento de dos preguntas orientadoras para los expertos, a las cuales cada uno debía responder en un tiempo establecido. Las preguntas fueron: ¿Qué elementos, situaciones o experiencias, destaca como tendencia contemporánea, en la gestión de organizaciones deportivas? ¿Qué retos se generan para las organizaciones deportivas del país, a partir de las tendencias actuales?

Las respuestas que los expertos dieron a las preguntas fueron grabadas en audio y luego transcritas en una hoja de cálculo de Excel donde fueron interpretadas y analizadas a partir de las categorías de interés. Este proceso de reducción de datos en categorías, se reconoce como codificación y se hizo desde las orientaciones de Marshall y Rossman (2010).

Como soporte teórico del proceso, se realizó una revisión de literatura con base en las orientaciones de Carrasco y Calderero (2007) y Cronin, Ryan y Coughlan (2008).

Finalmente, cuidando la validez de constructo, se desarrollaron procesos de retroalimentación con los expertos sobre el proceso investigativo.

\section{Resultados}

Se presentan los resultados a partir del análisis de contenido desarrollado con los expertos. Es importante tener presente algunas consideraciones:

Los resultados están agrupados por categorías que emergieron del análisis teórico y que, de alguna forma o componente, hicieron presencia también en el análisis de contenido que se hizo con las respuestas que aportaron los expertos. El orden de presentación no guarda relación con la frecuencia o importancia, dado que el objetivo no fue generalizar, sino más bien brindar una perspectiva de lo que piensan los expertos, por tanto, todas las respuestas fueron consideradas. En este sentido, se incluyen también algunas categorías que surgieron en el discurso de los expertos y que no se trataron en la literatura revisada.

En aras de mantener el anonimato de las personas que hicieron parte del análisis, se hará referencia a ellos como sujetos. En ocasiones se extraen algunas citas textuales de la transcripción de los informantes, para dar respaldo a lo expuesto. Para identificación, se utilizará la sigla Sa. Lx, donde "S" hace referencia a Sujeto, "a" es el número consecutivo asignado para diferenciarlos, "Lx" se refiere a la línea y número de ubicación de la transcripción que es citada.

\section{Innovación, marketing y nuevos modelos de negocio}

Los expertos expresan que la innovación, junto con la planificación, son considerados como una tendencia importante en la gestión de organizaciones, lo que implica dinamizar mercados, atender las demandas de la era digital que se vive y, ante todo, ser creativos para tomar decisiones de vanguardia " $d i$ namizar los mercados desde la creatividad que estamos buscando y meternos en un cuento que es la revolución digital y crear nuevos modelos de negocios" (S2. L2). Hoy las organizaciones no son estáticas, por tanto, sus estructuras deben ser cada vez 
más dinámicas, adaptando sus diseños organizacionales a las demandas que el entorno cambiante va haciendo: "las organizaciones nuestras tienen que tener una estructura dinámica (...) que puedan estar siempre al servicio y a disposición de las necesidades" (S3. L23)

\section{La contribución al bienestar de las comunidades}

Hay un interés marcado por generar estilos de vida saludables para las comunidades, teniendo en cuenta que hay cifras de más del $50 \%$ de la población deportiva inactiva (S2. L26). Existe una preocupación por recuperar lo que se ha denominado deporte de base y fortalecer la relación necesaria entre el deporte asociado y el deporte social comunitario. En este sentido, se expresa la necesidad de promover el deporte escolar como punto de partida del desarrollo deportivo de un país, propiciando la creación de clubes deportivos en las instituciones educativas para mantener a los niños activos, saludables y generar hábitos en ellos que permitan darle continuidad al sistema fortaleciendo a su vez, el deporte universitario desde otra perspectiva y no solo desde el alto rendimiento.

De igual manera, organizaciones deportivas del orden municipal y departamental en Medellín, vienen incorporando en sus agendas cada vez más, las prácticas y actividades que generen verdaderos impactos en las comunidades, en especial, en el tema social. Un verdadero reto es utilizar adecuada y eficazmente los recursos - generalmente insuficientespara responder y priorizar las demandas de los territorios. Se expresa la necesidad de generar ofertas individualizadas de acuerdo con cada realidad de los contextos en que se mueve la organización: "tenemos un reto grandísimo y es que tenemos que construir (...) una oferta diferente para cada comuna de acuerdo a su población, (...) al indice multidimensional de calidad de vida, (...) las necesidades básicas insatisfechas, (...) las problemáticas sociales (...)” (S3. L18)

Se plantean retos que tienen que ver con el aumento de cobertura para llegar a más sectores de la sociedad en la oferta de actividad física y deporte, generando un mayor impacto. Las organizaciones deportivas tienen un interés de lucro social y esto se logra cuando más personas del país participen de sus actividades: "nuestro gran reto es llegar a todas las comunidades de Colombia desde la actividad fisica, hacer un pais activo y saludable" (S1, L4). Un reto de esta dimensión, implica hacer una lectura permanente del contexto, en aras de reconocer las necesidades fundamentales de la población en tema de deporte y recreación y poder diseñar la oferta dando respuesta a ello: "ser capaces de hacer lecturas inteligentes de qué es lo que la comunidad necesita del deporte para llevarle lo que ella necesita" (S3, L7). Es necesario aclarar que las demandas de la comunidad deben estar acompañadas de una visión contextualizada de lo que es el deporte en el país, dado que la misma comunidad podría no reconocer cuál es su necesidad fundamental en este sentido.

El ocio es otro elemento que aparece y que está siendo reconocido como una actividad vital del ser humano. Para el caso de Antioquia, organizaciones que han atendido de manera primordial el alto rendimiento, dirigen la mirada a las actividades que promuevan el disfrute, la salud, el contacto con la naturaleza, la mejora de la calidad de vida y de la salud "estamos buscando algo más allá; es darle unas respuestas a las necesidades que hay en Antioquia frente al deporte, la actividad física, la recreación y lo que muchos no manejan hoy que es el ocio, hoy el ocio tiene que volverse una tendencia desde el punto de vista mundial (...), hoy el ocio hace parte de la vida del ser humano, y a eso le estamos apuntando más (...)" (S2. L3)

\section{La articulación de organizaciones del sector y el trabajo en redes}

Los expertos expresan la necesidad de que las organizaciones del sistema nacional del deporte realmente se articulen. El trabajo en redes es hoy una de las formas más valoradas que agrega valor no solo a la organización sino al sector mismo, por tanto, otras organizaciones pares no deben ser miradas como competencia, sino más bien como aliadas estratégicas para el logro de los objetivos sectoriales. De igual manera, las estructuras organizacionales rígidas y piramidales deben ser reemplazadas por estructuras flexibles, circulares, que dinamicen y agilicen los procesos y generen respuestas rápidas y oportunas: "estructuras que nos concentren más en las responsabilidades que en la jerarquias, la oportunidad de respuesta es fundamental para una entidad de servicio y desafortunadamente a veces entidades que tienen muchos niveles de consulta en la toma de decisiones, tienen un costo de oportunidad altísimo en la respuesta" (S3, L14).

Por otro lado, y pese a la resistencia que puede generar el tema, se habla de una relación necesaria del deporte con la política. Conviene participar en los escenarios donde se toman decisiones que pueden influir, positiva o negativamente en el deporte, dado que el sector no es autosuficiente y demanda recursos del Estado para su desarrollo: “¿En dónde se aprueban los presupuestos para el deporte? En los Concejos, en las Asambleas y en el Congreso de la República (...) tenemos que establecer unos vínculos con los Corporados para defender los intereses del deporte, eso si bajo un claro principio de neutralidad politica" (S3, L61)

\section{La formación de los dirigentes y la buena gobernanza}

La formación de los dirigentes deportivos del país es un tema que ha despertado el interés en académicos e investigadores. Los retos que se presentan en la gestión de organizaciones deportivas, demandan un perfil de dirigente con habilidades, 
conocimientos y actitudes para tomar decisiones adecuadas que conduzcan al desarrollo, sostenibilidad y posicionamiento de su organización: "la formación, capacitación y educación continua tienen que ser una exigencia permanente a quienes hagan parte de estas organizaciones" (S3, L25).

En relación con el tema de la formación del dirigente y las habilidades que debe desarrollar, se habla también de un reto interesante que tiene que ver con la gestión del conocimiento. Gestionar adecuadamente el conocimiento en la organización, significa que se reconoce el potencial de cada una de las personas que la integran, se utiliza adecuadamente en los procesos de la organización y se eleva al nivel de capacidades colectivas generando aprendizaje organizacional. Es un aspecto al que no se le ha dado mucha importancia y que resulta fundamental para la eficiencia organizacional: "gestionar el conocimiento es una tarea importantísima a la que de pronto (...) no le prestamos mucho interés y a veces a las personas las ubicamos en determinados cargos y les damos determinadas funciones y responsabilidades porque a ese le tocó, pero no porque tengamos identificado que es alli donde debe estar" (S3, L20).

Otra de las habilidades tiene que ver con la adecuada gestión de los recursos. En relación con los recursos económicos, por ejemplo, se reconoce que en el país los recursos para el deporte son insuficientes, por tanto, la habilidad del directivo consiste también en hacer un uso adecuado y óptimo de dichos recursos para lograr los impactos que realmente deban generarse.

En relación con la buena gobernanza, se habla de la ética deportiva como uno de sus principios. El comité olímpico internacional define claramente estos principios y aunque deberían ser ruta de trabajo en las organizaciones, es un tema todavía ausente que requiere un análisis profundo y al que hay que apostarle: "La transparencia, la integridad y la oposición a cualquier forma de corrupción (...) bajo esos principios nosotros tendriamos que comprometernos a gestionar nuestras organizaciones deportivas, pero desafortunadamente en nuestro medio yo siento que el tema de la buena gobernanza está siendo tratado más bien todavía desde un ámbito teórico" (S3, L43).

En este mismo sentido, uno de los retos que se señala como más importantes y preocupantes, es la mejora de la deteriorada imagen que tienen los dirigentes deportivos del país, producto de diversas actuaciones que han desencadenado una serie de escándalos y han dejado la imagen y reputación del deporte muy por debajo: “Cómo mejorar la deteriorada imagen que tenemos hoy por hoy los dirigentes deportivos? Ser dirigente deportivo en Colombia se está volviendo sinónimo de ser corrupto, recientes escándalos (...) colocan en este momento al deporte, yo diría casi que en cuidados intensivos frente a lo que tiene que ver con su imagen, credibilidad y la reputación de quiénes hacemos parte" (S3, L26). Estos hechos dan la idea de que la ética deportiva está en crisis, sin embargo, uno de los informantes señala que, por el con- trario, hay un resurgir de la ética en la sociedad actual que está cansada de tanta corrupción y que reclama y busca otras formas. Acciones en pro de ello pueden evidenciarse desde el movimiento olímpico que exige en cada comité olímpico, un código y un comité de ética que vele por su cumplimiento.

\section{TIC, redes sociales, e-sports}

Se identificó esta categoría en los hallazgos del análisis de contenido, desde la perspectiva de las nuevas tendencias. Los jóvenes de hoy muestran una tendencia hacia actividades que ubican en un segundo lugar a los tradicionales deportes como el fútbol, el baloncesto y el voleibol y es necesario que las organizaciones y las instituciones educativas, reconozcan y atiendan esta necesidad "Las nuevas tendencias deportivas nos están llamando constantemente a que repensemos el modelo deportivo colombiano (...) algo tenemos que hacer para no encerrarnos en la historicidad del deporte" (S2. L21). Los e-sports hacen parte de estas tendencias y deberán ser reconocidos por las organizaciones.

\section{Discusión}

A partir de las categorías previamente definidas, se hace la discusión triangulando los hallazgos de la literatura, con los hallazgos del análisis de contenido con los expertos. Desde el análisis de los autores, se incluyen otras categorías que se relacionan o emergen de lo hallado:

En relación con la categoría de la innovación, el marketing y nuevos modelos de negocio, Tortora (2018) resalta la necesidad de crear nuevos modelos de negocio, producto de las demandas que el entorno hace, específicamente, en relación con los estándares de calidad cada vez más altos para satisfacer las necesidades de los usuarios. Esto fue visible también desde la opinión de los expertos, quienes lo reconocen como una tendencia importante, sumado a la innovación y creatividad que se requieren para crear dichos modelos y dinamizar mercados.

Las Tecnologías de la Información y la Comunicación (TIC) son mencionadas por Bustelo-Ruesta y GarcíaMorales-Huidobro (2001) y Herrera (2008) como una de las causas de los retos que hoy se generan en la organizaciones y las redes sociales uno de los principales medios de comunicación en la sociedad (López-Carril, Villamón et al., 2019). En el análisis de contenido, se reconoce que las organizaciones deportivas están llamadas a atender las demandas de la revolución digital que hoy se vive, lo que implica la adecuación o implementación de unos recursos humanos y tecnológicos que permitan comunicar lo que la organización hace, intercambiar información con usuarios, hacer lecturas del entorno y, en definitiva, lograr visibilidad y reconocimiento. 
En cuanto a la contribución del deporte y la actividad física al bienestar de las comunidades desde lo social, lo económico y la salud, Zeimers et al. (2019) advierten sobre las tensiones que se van generando entre el deporte de élite y el deporte para todos. En Colombia, este hecho también se hace visible y se evidenció en el análisis de contenido desde la apuesta que las organizaciones deportivas del orden municipal, departamental y nacional, hacen por un deporte que llegue a cada vez más sectores de la población, esto es, un deporte social - comunitario que genere verdaderos impactos en la salud de las personas.

De otro lado, llama la atención en diversos estudios, entre ellos el de Padierna et al. (2019), el perfil de formación de los dirigentes del país, dado que no solo desde la gestión de instalaciones deportivas, sino también desde otro tipo de organizaciones (Cardona, Padierna, Córdoba y González, 2017; Escobar, 2011), las personas que ocupan estos cargos, especialmente en lo público, no cuentan con formación específica ni complementaria en administración, siendo esto un asunto que debe ser atendido de manera urgente, no solo por las instituciones formadoras, sino también por los organismos de gobierno en aras de fortalecer las políticas, normas y leyes que regulen los cargos directivos en las organizaciones deportivas del país, desde elementos conceptuales, procedimentales y actitudinales que obliguen a que las personas que allí se desempeñen cuenten con la preparación y formación adecuadas. Esto se hizo evidente también en los resultados, mencionando la formación permanente del recurso humano, como un aspecto importante que debe incentivarse al interior de las organizaciones.

A propósito de la formación, siendo éste uno de los temas que más ha despertado el interés de los autores por las condiciones ya mencionadas en Colombia, se considera que un dirigente debe dominar las teorías de la organización y los modelos de gestión. En este sentido, se ha mencionado desde Fernández-Alles y Valle-Cabrera (2006) y Cardona (2017) que las instituciones y organizaciones deportivas tendrían que funcionar de la misma manera que cualquier otra organización y que, por tanto, las teorías y principios que se han desarrollado en el estudio de las organizaciones, son aplicables a ellas. Aunque probablemente no sea de manera intencionada, algunos de los principios y teorías que se han desarrollado en el estudio de las organizaciones, se hicieron visibles en los resultados. Se mencionan a continuación:

- La planeación: como fase dentro de la teoría del proceso administrativo, la planeación es uno de los aspectos de suma importancia en la gestión de organizaciones, dado que posibilita tener claridad sobre los objetivos organizacionales y los cursos de acción para conseguirlos. Junto con la innovación se mostró como una tendencia importante, lo que hace referencia a la habilidad que deben tener los directivos para ser creativos en sus diseños organizacionales, dinamizando la organización y generando ventaja competitiva.

- El análisis del entorno: este componente del diagnóstico organizacional, es un elemento que cotidianamente debe hacer presencia en las organizaciones. Hacer lectura del entorno interno y externo, es justamente lo que permitirá identificar tendencias que hoy deben incorporarse en los planes institucionales como el ocio, el deporte escolar, los deportes extremos, entre otros, por supuesto, con el respectivo análisis de contexto de la propia organización.

- Recursos y capacidades: todas las actividades que demanda una organización, tienen unos recursos implicados. En los resultados se menciona como uno de los retos, la utilización adecuada y eficaz de los recursos para responder a las demandas que el entorno hace, lo cual coincide con lo señalado en la literatura por Barney (2001) citado por Cardona (2017) como una de las habilidades de los directivos para generar aprendizaje organizacional y ventaja competitiva.

Se hace aquí una invitación a los directivos, para que respalden sus acciones a partir de las orientaciones que se han desarrollado desde las teorías de la organización. Ellas, en sí mismas, actúan como modelos de gestión en las organizaciones y aportan fundamentación teórica al quehacer como directivo, por tanto, deberían estar siempre presente.

Otro tema, tal vez de aparición reciente en el contexto colombiano, tiene que ver con la gestión del conocimiento, que se menciona también como reto importante, reconociendo que es algo a lo que posiblemente no se le ha prestado mucha atención. La literatura señala que el conocimiento es el recurso intangible más valioso que tiene una organización, por tanto, debe ser gestionado adecuadamente para que no se quede en una habilidad individual, sino que se eleve a capacidad organizacional. Esto debería ser también un componente en la formación de dirigentes deportivos.

Otra de las tendencias y retos que se mencionan también en los resultados, tienen que ver con el trabajo en redes. Se expresa la necesidad de establecer alianzas y trabajar de manera colaborativa. Hoy las organizaciones pares no deben mirarse como competencia, sino más bien como un posible aliado estratégico que posibilitará el crecimiento de la organización y del campo organizacional. Este es uno de los retos que también expresa el estudio de Universitat Pompeu Fabra (2017).

\section{La Gestión del cambio organizacional}

Se incluye esta categoría como emergente, dado que los retos indican la necesidad de incorporar cambios en las organizaciones. La literatura seńala que el cambio es un acontecimiento 
permanente, producto de la globalización y el auge que vienen teniendo las TIC (Bustelo-Ruesta y García-Morales-Huidobro, 2001; Cardona, 2017). En los resultados se hizo evidente que uno de los principales retos que tienen las organizaciones es adaptar sus diseños organizacionales a nuevas demandas del entorno y entre ellas, se menciona justamente la dinamización de mercados -que puede ser concebida como una forma de globalización- y la revolución digital. En este sentido, es necesario que las organizaciones, reconociendo esta necesidad de adaptación y respuesta al cambio, incorporen en sus prácticas organizaciones, rutas o modelos de gestión del cambio organizacional para que puedan adelantar estos procesos de manera exitosa. Llama la atención que, pese a esta necesidad, no se menciona en los discursos de los informantes, estrategias para adelantar eficazmente el cambio en sus organizaciones. Conviene retomar trabajos como los de Lewin (1988), Kotter y Schlesinger (2008) y Cardona (2017) que orientan sobre las acciones que debieran generarse cuando se adelanta un cambio en la organización, que seguramente permitirán con mayor respaldo, que el cambio sea sostenible, que implique realmente mejora organizacional y, en especial, que sea aceptado por todos los actores lo cual se logra con un cuidadoso trabajo de preparación, en el que se reduzcan las resistencias que de manera inevitable se presentan.

\section{Conclusiones}

Este trabajo hace una contribución al área de la administración y gestión deportiva, presentando una perspectiva frente a las tendencias y retos que se presentan hoy en la dirección de organizaciones deportivas. A partir de los hallazgos del estudio, se concluye que las organizaciones deportivas están en constante cambio, asumiendo una serie de retos que surgen del análisis permanente que hacen de su entorno. La gestión del cambio organizacional cumple entonces un papel fundamental y entra a delinearse como una de las habilidades que debe dominar el directivo de la organización para llevar a feliz término sus nuevos proyectos y procesos.

Otro de los principales retos tiene que ver con la capacidad de hacer adecuados diagnósticos organizacionales que permitan tener una visión clara y objetiva el entorno interno y externo y, a partir de allí, proponer, orientar, o bien re diseñar procesos y estructuras administrativas que, en todo caso, permitan el logro de los objetivos organizacionales. Importante señalar aquí que el análisis de tendencias, como cursos de acción, demanda de la habilidad del directivo para identificar lo que realmente es útil y aplicable a su organización, toda vez que en ocasiones puede no contarse con los recursos y capacidades necesarios o bien, puede no ser adecuado para el contexto de la organización.

Seguido del diagnóstico organizacional, vienen los planes de la organización y como tendencia, se ponen en escena asuntos de innovación y creatividad, es decir, hoy la planeación trasciende aquellas formas estáticas e intenta dinamizar la organización hacia otros ámbitos y contextos en el orden no solo local, sino también nacional e internacional e involucrando de manera cada vez más creciente, el uso de tecnologías de la información y la comunicación.

Se evidencia también una apuesta por diversificar la oferta de servicios dando espacio a otras actividades que vienen surgiendo como los deportes extremos, los e-sports y otras actividades que contribuyen al uso adecuado el ocio y el tiempo libre. Importante también abarcar cada vez más sectores de la sociedad.

Temas como la gestión del conocimiento y la buena gobernanza empiezan a tomar fuerza en el contexto colombiano. Se invita a identificar y potenciar el conocimiento que tienen las personas que hacen parte de la organización con lo cual se beneficia el empleado desde la perspectiva de la motivación y la satisfacción laboral, y la organización desde el aprendizaje que se genera. En relación con la buena gobernanza, fortalecer y mostrar las buenas prácticas administrativas es tema de interés nacional. Aunque esto es un deber ser del dirigente, vienen desarrollándose una serie de acciones que permitirán generar modelos de gestión en este sentido, como por ejemplo la investigación que se viene desarrollando a nivel nacional con el respaldo del Comité Olímpico Colombiano, sobre gobernanza y legitimidad de las federaciones deportivas colombianas, lo cual seguramente aportará al posicionamiento de una buena imagen de país.

Finalmente, es claro que hay una demanda creciente por contar con perfiles de formación relacionados con la administración de organizaciones en aras de fortalecer las acciones que desde allí se generan y facilitar el desarrollo y consecución de los retos que se presentan. Esto es una implicación para directivos de gobierno y de instituciones de educación superior.

\section{Limitaciones y futuras líneas de investigación}

Se presenta un panorama frente a las tendencias y retos en la gestión de organizaciones deportivas, sin embargo, no es posible generalizar los resultados dado que se contó únicamente con la opinión de tres expertos. Esto constituye un punto de partida para futuros estudios en otros ámbitos y contextos.

\section{Referencias}

1. Barney, J. (2001). Resource-based theories of competitive advantage: A ten- year retrospective on the resource-based view. Journal of Management, 27, 643-650.
2. Bustelo-Ruesta, C., y García-Morales-Huidobro, E. (2001). Tendencias en la gestión de la información, la documentación y el conocimiento en las organizaciones. El Profesional de La Información, 10(12), 4-7. 
3. Cardona, L., Padierna, J., Córdoba, M., González, E. (2013). Fundamentos conceptuales en torno al área de la administración deportiva. Revista Educación Física y Deporte, 32(2), 1471-1478. https://doi.org/ http://aprendeenlinea.udea.edu.co/revistas/index.php/educacionfisicaydeporte/article/view/17899

4. Cardona, L., Padierna, J., Córdoba, M. y González, E. (2017). Características y demandas administrativas de las organizaciones deportivas del municipio de Medellín. Revista Ímpetus, 11(2), 61-72. https://doi.org/ http://idiomas.unillanos.edu.co/revista_impetus/vol11_n2/mobile/index.html\#p=3

5. Cardona, L. (2017). El cambio organizativo en las instituciones de educación superior. Universidad de Valencia. Retrieved from http://roderic. uv.es/handle/10550/61016

6. Cardona, L. y Padierna, J. (2017). Administración y gestión de organizaciones deportivas. Medellín: Editorial Universidad de Antioquia.

7. Cardona, L. (2013). Línea de maestría en administración y gestión deportiva: Una nueva puerta hacia la producción de conocimiento. VIREF Revista de Educación Física, 2(3). https://doi.org/http://aprendeenlinea. udea.edu.co/revistas/index.php/viref/article/viewFile/16015/13884

8. Carrasco, J. y Calderero, J. (2007). Aprendo a investigar en educación (2a.). Madrid - España: RIALP S.A.

9. Casassus, J. (2000). Problemas de la gestión educativa en América Latina. UNESCO.

10. Cronin, P., Ryan, F. y Coughlan, M. (2008). Undertaking a Literature Review: a step-by-step approach. British Journal of Nursing, 17(1). https://doi.org/10.1177/107808747000500401

11. Da Pena A, Fierli E. (2017). Actas Congrés Esport Local. In: La Marie va de visita: guia il. lustrada del recinte modernista de Sant Pau. Barcelona: Recinto Modernista de Sant Pau.

12. Escobar, J. (2011). Retos y estrategias para la administración deportiva en Colombia. EFDeportes. Com, Revista Digital, 15(152), 1-7.

13. Fernández-Alles, M. y Valle-Cabrera, R. (2006). Reconciling institutional theory with organizational theories. How neoinstitutionalism resolves five paradoxes. Journal of Organizational Change Management, 19(4), 503-517. https://doi.org/10.1108/09534810610676699

14. Herrera, B. (2008). Tendencias mundiales de la investigación en el área de gestión de organizaciones. Teknos. Revista Cientifica, 4(2).

15. Kotter, J., y Schlesinger, L. (2008). Choosing Strategies for Change.
Harvard Business Review, July/ Augu(July 2008), 1-8. https://doi.org/ Article

16. Krippendorff, K. (1990). Metodología de análisis de contenido. Teoría y práctica. Barcelona: Paidós.

17. Lewin, K. (1988). La Teoría del Campo en la Ciencia Social. Barcelona: Ediciones PAIDOS

18. López-Carril, S., Añó, V. y Villamón, M. (2019). El campo académico de la gestión del deporte: pasado, presente y futuro. Cultura, Ciencia, Deporte, 14(42), 277-287. https://doi.org/10.1017/ CBO9781107415324.004

19. López-Carril, S., Villamón, M. y Añó, V. (2019). Conceptualización de los medios sociales: oportunidades para la gestión del deporte. Retos, 36(July), 578-583.

20. López, M. (2015). The intelligence of the trends. Universidad de Murcia. Retrieved from https://www.tdx.cat/bitstream/handle/10803/371449/ TMJLV.pdf

21. Marshall, C. y Rossman, G. (2010). Designing Qualitative Research (Second edi). Sage Publications.

22. Ogburn, W. (1957). Tendencias sociales. Sociology and Social Research, XLII, 211-217.

23. Padierna, J., Cardona, L., Córdoba, M., Giraldo, V. y Colorado, A. (2019). Modelos de dirección en la gestión de instalaciones deportivas. Revista Peruana de Ciencias de La Actividad Física y Del Deporte, 6(4), 810-820.

24. Pires, A. y Machado, V. (2006). Gestión por Procesos en el Diseńo de las Organizaciones. Información Tecnológica, 17(1), 1-12.

25. Tortora, M. (2018). Sport Management and Sustainability Innovation Challenges. In Sports media, marketing and management: breakthroughs in research and practice (pp. 301-313). IGI Global. https://oi org/10.4018/978-1-5225-5475-2.ch017

26. Universitat Pompeu Fabra. Barcelona school of management. (2017). Cinco retos de la industria del deporte para 2018.

27. Zaratiegui, J. (1999). La gestión por procesos: su papel e importancia en la empresa. Economía Industrial, 330.

28. Zeimers, G., Shilbury, D., Willem, A. y Zintz, T. (2019). Hybridity in Non-profit Sport Organisations : Organisational Challenges. New Orleans: North American Society for Sport Management Conference (NASSM 2019). 